\title{
Optimal tight glycaemic control supported by differential geometric methods
}

\author{
L. Kovács ${ }^{1}$, P. Szalay ${ }^{1}$, B. Benyó ${ }^{1}$ and J.G. Chase ${ }^{2}$ \\ ${ }^{1}$ Budapest University of Technology and Economics, Dept. of Control Engineering and Information Technology, Budapest, Hungary \\ ${ }^{2}$ University of Canterbury, Dept. of Mechanical Engineering, Centre for Bio-Engineering, Christchurch, New Zealand
}

\begin{abstract}
Optimal control of an Intensive Care Unit (ICU) metabolic model from nonlinear control point of view is presented in the current paper. The transformation of a clinically validated nonlinear model into a series of integrators via exact linearization and asymptotic output tracking is performed. Both methods need the value of the state variables; therefore Kalman-filter extended for nonlinear systems is applied. Finally, linear optimal LQ method is applied on the ICU model handled with differential geometric approach. Results are demonstrated on input data recorded in actual clinical environment.
\end{abstract}

Keywords - Exact linearization, ICU model, asymptotic tracking, Kalman-filtering, LQ control.

\section{INTRODUCTION}

Critically ill patients admitted to the ICU often display hyperglycaemia and insulin resistance associated with adverse outcomes, which can result in increased morbidity and mortality [1]. Tight glycaemic control (TGC) can reduce these adverse outcomes [2], [3], as well as reducing economic costs [4]. Hence, TGC using model-based methods has become an active research field [5]. The best known model is the minimal model of Bergman [6]. However, the model's simplicity is a disadvantage, as significant components of glucose-insulin interaction are neglected. Consequently, different models were derived from the minimal model, trying to generalize / extend the validity for the ICU case [7], [8], [9]. The nonlinearity in each of the models represents specific control aspects, but the applied control strategies are usually developed for their linearized (i.e. working point based) versions.

The current paper investigates this aspect in terms of differential geometric approach [10]. Exact linearization can be applied by corresponding coordinate transformation. Hence, the validity of linear controllers can be extended from the neighborhood of a working point to a larger subset of the state-space bounded by specific constraints. This approach might not increase the performance of the controllers to a great extent, but the reliability of tight glucose control can directly affect the quality of life of an ICU patient. The applied methodology is combined with optimal LQ method and the efficiency of the controller is demonstrated on real input data recorded in actual clinical environment.

\section{ICU MODEL}

The clinically validated model of [7] is basically a generalization of the Bergman minimal model [6]. It better captures insulin losses to the liver and kidneys, and saturation dynamics through the use of Michaelis-Menten functions. The parameters of the model have been identified to a wide range of patients. Below, we summarize the model equations. Numerical values can be found in [7].

$$
\begin{aligned}
\dot{G}(t) & =-p_{G} G(t)-S_{I}\left(G(t)+G_{E}\right) \frac{Q(t)}{1+\alpha_{G} Q(t)} \\
\dot{Q}(t) & =-n_{C} Q(t)+\frac{n_{I}}{V_{Q}}(I(t)-Q(t)) \\
\dot{I}(t) & =-n_{K} I(t)-\frac{n_{L} I(t)}{1+\alpha_{I} I(t)}-\frac{n_{I}}{V_{P}}(I(t)-Q(t)) \\
& +\left(1-x_{L}\right) \frac{u_{e n}}{V_{P}}+\frac{u_{e x}(t)}{V_{P}}
\end{aligned}
$$

The states of the system are:

- $G$ is the deviation of plasma glucose concentration (mmol/L) from equilibrium level $\left(G_{E}\right)$;

- I represent the concentration of plasma insulin resulting from external input (mU/L);

- $Q$ is the concentration of insulin bounded to interstitial sites (mU/L);

Regarding the inputs of the system, $P$ represents the glucose input through enteral feeding ( $\mathrm{mmol} / \mathrm{min}$ ), while $u_{e x}$ is the intravenously administered external insulin ( $\mathrm{mU} / \mathrm{min}$ ).

Regarding system-parameters, $p_{G}$ is the endogenous glucose clearance; $S_{I}$ the insulin sensitivity; $\alpha_{G}$ the insulin dependent glucose clearance/insulin effect; $E G P$ the endogenous glucose production; $V_{G}, V_{Q}$ and $V_{P}$ glucose distribution volume, interstitial fluid volume and plasma volume with fast exchanging tissues; $x_{L}$ the fraction of hepatic extraction; $n_{K}, n_{L}$ kidney and liver clearance rates of plasma insulin; $n_{I}$ diffusion constant of insulin between compartments; $n_{C}$ cellular insulin clearance rate from interstitium; $\alpha_{I}$ plasma insulin disappearance rate; $u_{e n}$ constant endogenous insulin production. Detailed description can be found in [7]. 


\section{Methods}

\section{A. Exact linearization via feedback}

The concept of exact linearization of a nonlinear system via nonlinear state feedback control was introduced in [10]. Consider a SISO nonlinear system in the form:

$$
\begin{aligned}
& \dot{x}(t)=f(x(t))+g(x(t)) u(t) \\
& y(t)=h(x(t))
\end{aligned}
$$

$f$ and $g$ are smooth $R^{n}$-valued mappings and $h$ is a smooth real-valued mapping defined on an open set $U \subset R^{n}$. This system is said to have a relative degree $r$ on an open and dense subset $V$ of the open set $U$ if for all $x_{0} \in V \subset U:$

- $\quad L_{g} L_{f}^{k} h(x)=0$ for all $x$ in a neighbourhood of $x_{0}$ and all $k<r-1$;

- $L_{g} L_{f}^{r-1} h\left(x_{0}\right) \neq 0$.

where $L_{f} h(x(t))$ is the Lie-derivate of $h(x(t))$ along $f$ [10]. Let us assume that the nonlinear system (2) has relative degree $r=\operatorname{dim}(x)=n$ at $x_{0}$. If for the coordinate mapping:

$z_{1}=\Phi_{1}(x)=h(x)$

$z_{2}=\Phi_{2}(x)=L_{f} h(x)$

:

$z_{r}=\Phi_{r}(x)=L_{f}^{r-1} h(x)$

$\Phi(x)=\left(\begin{array}{lll}\Phi_{1}(x) & \ldots & \Phi_{n}(x)\end{array}\right)^{T}$ has a nonsingular Jacobian matrix at $x_{0}$ and qualifies as local diffeomorphism in a neighborhood of $x_{0}$. Therefor, we can transform the nonlinear system into a series of $n$ integrators with the following control law:

$u(t)=\frac{1}{L_{g} L_{f}^{n-1} h\left(\Phi^{-1}(z)\right)}\left(-L_{f}^{n} h\left(\Phi^{-1}(z)\right)+v(t)\right)$

\section{B. Asymptotic Output Tracking}

Instead of forcing the output of a nonlinear system to follow exactly the output of a prescribed reference linear system, it is more realistic to produce an output that, irrespectively of the initial state of the system converges asymptotically to the output of a reference linear system, which is called asymptotic output tracking [10]. Let us have a reference linear system of the form:

$\dot{\varsigma}=A \cdot \varsigma+B \cdot w$

$y_{R}=C \cdot \varsigma$

Choose this system with a relative degree equal to or higher than the relative degree of the nonlinear system:

$y_{R}^{(i)}(t)=C A^{i} \varsigma(t), 0 \leq i \leq m-1, m \geq r$

$y_{R}^{(m)}(t)=C A^{m} \varsigma(t)+C A^{m-1} B w$

If we have an appropriately chosen $r$-th order asymptotically stable linear system with the transfer function:

$$
H(s)=\frac{y(s)}{y_{r}(s)}=\frac{b_{r} s^{r}+b_{r-1} s^{r-1}+\cdots+b_{0}}{s^{r}+a_{r-1} s^{r-1}+\cdots+a_{0}}
$$

then using the control law:

$$
\begin{gathered}
u(t)=\frac{1}{L_{g} L_{f}^{r-1} h(z(t))}\left(-L_{f}^{r} h(z(t))-\sum_{i=0}^{r-1}\left(a_{i} \cdot L_{f}^{i} h(z(t))\right)+\right. \\
\left.+b_{r} \cdot C A^{r-1} B w(t)+C \sum_{j=0}^{r}\left(b_{r} \cdot A^{i}\right),(t)\right)
\end{gathered}
$$

we can force the nonlinear system to have the same output for the input $w$ as the series of the linear system (5) and (7).

If the relative degree of the linear system (5) is higher than the relative degree of the nonlinear system, then $b_{r} C A^{r-1} B w(t)=0$.

\section{Kalman-filter}

The main idea of the Kalman-filter is that from the inputs and the outputs of a linear system with known dynamics computes a prediction of the state variables and adds a correction to this prediction based on the deviation of the measured and estimated output [11].

In our case, both exact linearization and asymptotic output tracking need the values of the state variables. However, in practice the only measured quantity is either the intravenous or subcutaneous glucose concentration. The sensors used in these measurements usually have relatively high noise and a sampling time of 3-5 minutes; therefore, a Kalman-filter is needed to provide adequate state-estimation.

Kalman-filtering has a wide literature. In our case, the algorithm presented in [11] was used. 


\section{Methods}

The relative degree of model (1) is equal to the number of states; therefore, the coordinate transformation for both exact linearization and asymptotic output tracking are unequivocally determined. The system has a single output, and as only the intravenously administered external insulin input $\left(u_{e x}\right)$ can be controlled, the system can be considered as a SISO system. The other input $(P)$ can be regarded as a known time-varying parameter. Considering the connections between each compartment, the system can be divided into a subsystem described by last two differential equations of (1) with $Q$ as its output, and another subsystem described by a single differential equation (the first equation of (1)). Hence, it is possible to determine separately the control law for exact linearization and asymptotic output tracking.

The first subsystem is transformed into a series of integrators through exact linearization. The local coordinate transformation determined by the Lie-derivates is a local diffeomorphism regardless of the state variables.

$$
\begin{aligned}
& z_{1}=\Phi_{1}(x)=x_{2} \\
& z_{2}=\Phi_{2}(x)=-n_{c} x_{2}+\frac{n_{I}}{V_{Q}}\left(x_{3}-x_{2}\right) \\
& \left|\frac{\partial \Phi}{\partial x}\right|=\left|-\left(n_{c}+\frac{n_{I}}{V_{Q}}\right) \quad \frac{n_{I}}{V_{Q}}\right|=\frac{n_{I}}{V_{Q}}
\end{aligned}
$$

The control law (4) is applicable as long as $-\alpha_{I}^{-1} \neq x_{3}$. The second control law of the controller realizes asymptotic output tracking, and is working with the following nonlinear system:

$$
\begin{aligned}
\dot{x}_{1}(t)= & -p_{G} x_{1}(t)-S_{I}\left(x_{1}(t)+G_{E}\right) \frac{x_{2}(t)}{1+\alpha_{G} x_{2}(t)} \\
& +E G P+\frac{P(t)}{V_{G}} \\
\dot{x}_{2}(t) & =x_{3}(t) \\
\dot{x}_{3}(t) & =u(t)
\end{aligned}
$$

The local coordinate-transformation for this system is a local diffeomorphism as long as:

$$
\left|\frac{\partial \Phi(x)}{\partial x}\right|=\left(-\frac{S_{I}\left(x_{1}+G_{E}\right)}{\left(1+\alpha_{G} x_{2}(t)\right)^{2}}\right)^{2} \neq 0
$$

The properties of the second control loop are determined in a way that the nonlinear system would track the output of its own steady-state linearization with appropriately fast error dynamics.

To provide the values of state variables for the control laws, an extended Kalman-filter algorithm has been used. The output signal was measured with 5 minute sampling time. The performance of the Kalman-filter is presented in Fig. 1.

It is also possible, to use exact linearization on the system in the second loop as well and then apply LQ control [11], on the resulting series of integrators. In this case, the following quadratic cost-function can be used:

$$
J(x, u)=\frac{1}{2} \int_{0}^{\infty}\left(y^{2}+50 u^{2} d t\right)
$$

Consequently, the following state-feedback vector is obtained:

$$
K=\left(\begin{array}{lll}
0.1414 & 0.5429 & 1.0420
\end{array}\right)
$$

The output of the nonlinear system with and without using the presented control laws is compared with the series of linear systems determined for the tracking error (7) and the linear system (5) that needs to be followed (Fig. 2).

For the steady-state linearization of (1) a classical PID controller was implemented to show the advantages of the applied methodology even in case of a low level controller.

Fig. 3 presents the results of it in case of glucose input profile recorded in real clinical environment. It can be seen that quality parameters (settling time and overshoot) gave better results in case of asymptotic tracking then in case of controlling the nonlinear model directly. The structure of the controller was first determined in continuous time domain, and then transformed into a discrete-time as follows:
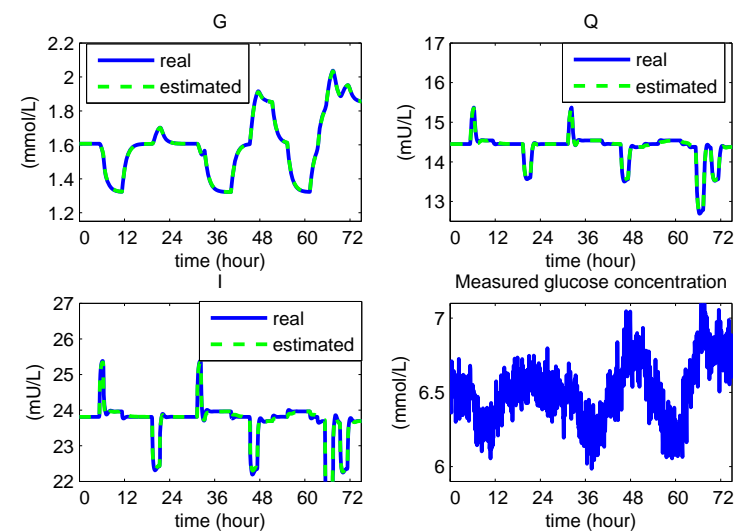

Fig. 1 The real and estimated values of state variables acquired from the simulated output samples with added Gaussian measurement noise 


$$
\begin{aligned}
& W_{P I D}(s)=A_{p} \frac{\left(T_{I} s+1\right)}{T_{I} s} \frac{\left(T_{D} s+1\right)}{\left(\left(T_{D} / n\right) s+1\right)} \\
& D_{P I D}(z)=\left(1-z^{-1}\right) Z\left\{L^{-1}\left\{W_{P I D}(s)\right\}\right\}
\end{aligned}
$$

The use of LQ control instead of classical PID clearly presents (Fig. 4) that combination of the nonlinear methodology applied together with linear optimal control can give good performance in the TGC problem in ICU.

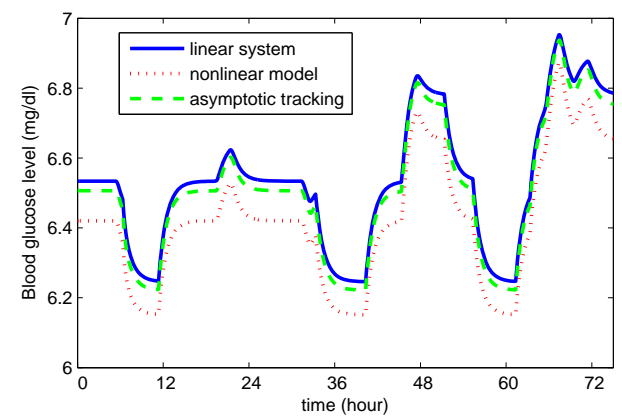

Fig. 2 Comparison of the steady-state linearization, asymptotic output tracking and original model output in case of model (1).
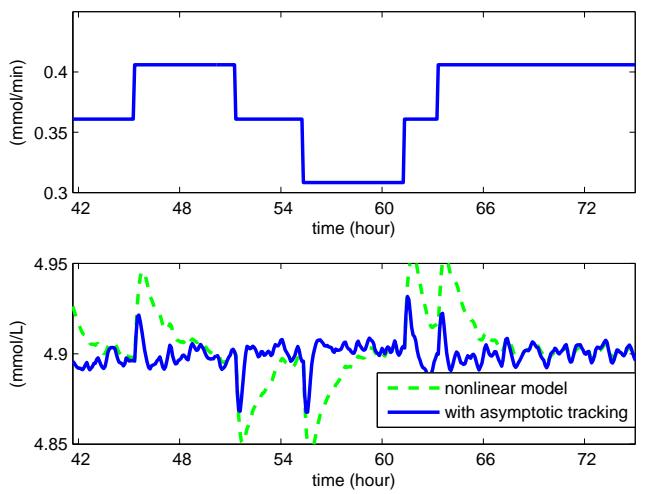

Fig. 3 PID controller performance with and without asymptotic output tracking (bottom) in case of glucose absorption profile taken from real clinical environment (top).

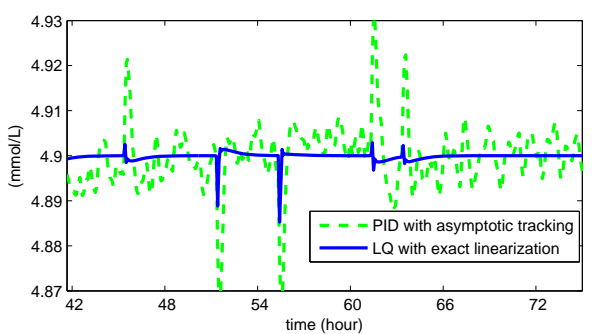

Fig. 4 Comparison of PID control using asymptotic tracking with LQ control using exact linearization.

\section{CONCLUSIONS}

Optimal control of a clinically validated ICU metabolic model from nonlinear control point of view is presented in the current paper. It was demonstrated that combination of the nonlinear methodology applied together with linear optimal control can give good performance in the TGC problem in ICU instead of pure linear model based control.

\section{ACKNOWLEDGMENT}

This work was supported in part by Hungarian National Scientific Research Foundation, Grants No. OTKA 82066, CK80316. It is connected to the scientific program of the "Development of quality-oriented and harmonized $\mathrm{R}+\mathrm{D}+\mathrm{I}$ strategy and functional model at BME” project, supported by the New Hungary Development Plan (Project ID: TÁMOP-4.2.1/B-09/1/KMR-2010-0002).

\section{REFERENCES}

1. Capes SE, Hunt D, Malmberg K et al. (2000) Stress hyperglycaemia and increased risk of death after myocardial infarction in patients with and without diabetes: a systematic overview. Lancet 355(9206):773778.

2. Van den Berghe G, Wouters P, Weekers F et al. (2001) Intensive insulin therapy in the critically ill patients, $\mathrm{N}$ Engl $\mathrm{J}$ Med 345(19):1359-1367.

3. Chase J, Shaw GM, Le Compte A et al. (2008) Implementation and evaluation of the SPRINT protocol for tight glycaemic control in critically ill patients: a clinical practice change. Crit Care 12(R49).

4. Van den Berghe G, Wouters PJ, Kesteloot K et al. (2006). Analysis of healthcare resource utilization with intensive insulin therapy in critically ill patients. Crit Care Med 34(3):612-616.

5. Chase J, Shaw GM, Wong XW et al. (2006) Model-based Glycaemic Control in Critical Care - A review of the state of the possible. Biomed Signal Proces \& Control 1(1):3-21.

6. Bergman RN, Philips LS, Cobelli C (1981) Physiologic evaluation of factors controlling glucose tolerance in man. J Clin Invest 68:14561467.

7. Lotz TF, Chase JG,. McAuley KA et al. (2008) Monte Carlo analysis of a new model-based method for insulin sensitivity testing. Comput Meth Progr Biomed 89:215-225.

8. Wong XW, Chase JG, Shaw GM et al. (2006) Model predictive glycaemic regulation in critical illness using insulin and nutrition input: A pilot study. Med Eng \& Phys 28:665-681.

9. Van Herpe T, Espinoza M, Haverbeke N et al. (2007) Glycemia Prediction in Critically Ill Patients Using an Adaptive Modeling Approach. J Diab Sci \& Techn 1(3):348-356.

10. Isidori A (1995) Nonlinear Control Systems. 3rd ed., Springer, Berlin.

11. Lantos B (2003) Theory and design of control systems II. (in Hungarian), Akademia Press, Budapest.

Author: Levente Kovács

Institute: Budapest University of Technology and Economics

Street: $\quad$ Magyar tudósok krt. 2.

City: Budapest

Country: Hungary

Email: lkovacs@iit.bme.hu 